\title{
Emil Behring. In memoriam
}

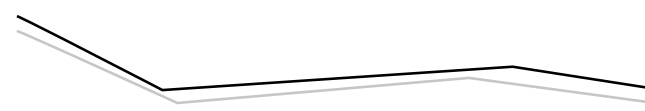

Eva María Salinas Miralles*凶

Salinas Miralles, E. M. (2017). Emil Behring. In memoriam [Obituario]. Investigación y Ciencia de la Universidad Autónoma de Aguascalientes, 25(72), 129-130.

En el centenario de su muerte, no podemos dejar de recordar la vida y obra de Emil Behring, científico considerado el fundador de la inmunología moderna. Nació el 15 de marzo de 1854 en Hansdorf (en la antigua Prusia) y falleció el 31 de marzo de 1917 en Marburg (Alemania). Miembro de una familia modesta, fue el mayor de 13 hermanos. Sus padres no pudieron hacer frente a sus estudios universitarios, pero sus maestros lo apoyaron para conseguir una beca y estudiar en la Escuela de Medicina de la Armada en Berlín, lo que lo comprometió a prestar apoyo al ejército durante varios años después de obtener su grado en 1880. Sin embargo, durante su estadía como médico militar en Polonia encontró tiempo para iniciar sus trabajos de investigación sobre los efectos antisépticos y antitóxicos del iodo y sus derivados. Rápidamente sobresalió en el ambiente científico y fue traslado a Berlín, al Instituto de Higiene liderado por Robert Koch.

Las circunstancias sociales de finales del siglo XIX marcaron de manera importante la carrera de Emil Behring. En aquella época una epidemia de difteria azotaba Europa causando miles de muertes en la población civil y afectando principalmente a los niños. En el impero alemán, 50,000 niños morían cada año de esta enfermedad. En este escenario, Emil Behring desarrolló su carrera científica y consiguió logros que cambiaron el rumbo de la medicina y la inmunología. En 1890, publicó una serie de artículos en los que describió lo que sería la cura para la difteria. En colaboración con el médico japonés Shibasaburo Kitasato, demostró cómo la inoculación de animales con cultivos estériles de difteria causaba que los animales produjeran antitoxinas (anticuerpos) en su sangre. Además, demostró que la inyección de

\footnotetext{
* Departamento de Microbiología, Centro de Ciencias Básicas, Universidad Autónoma de Aguascalientes. Av. Universidad 940, Ciudad Universitaria, C. P. 20131, Aguascalientes, Aguascalientes, México. Correo electrónico: emsalin@correo.vaa.mx

Autor para correspondencia
}

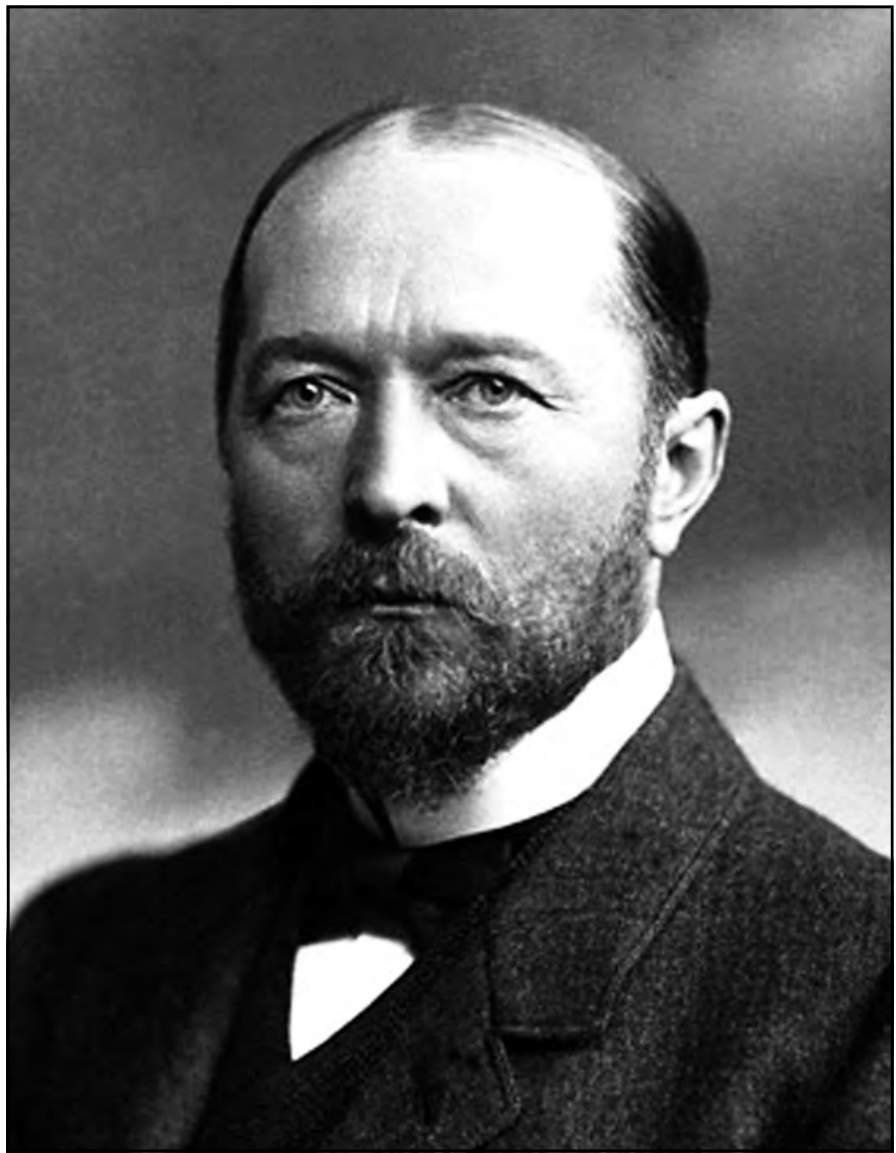

Emil Behring.

Imagen tomada de Nobel Prize.org.

las antitoxinas generadas en un animal podía curar a otro animal enfermo de difteria. En 1891, inyectó por primera vez antitoxinas en un niño de 8 años de edad gravemente enfermo de difteria, logrando su recuperación. Posteriormente, su trabajo se centró en la fabricación del antisuero (con antitoxinas) en grandes cantidades y en 1893 inició las pruebas clínicas. Un año después publicó los resultados mostrando una eficacia de $77 \%$ en la cura de la difteria. El antisuero fue aprobado para tratar la 
IIVESTIGACIÓn Y CIERCIA DE LA UNIVERSIDAD AUTÓNOMA

130 difteria y en un año se redujo a la mitad la tasa de mortalidad por la enfermedad en Alemania.

Emil Behring contrajo matrimonio en 1896 con Else Spinola, una jovencita de 20 años, hija del director del hospital de Charité en Berlin y con la que tuvo siete hijos. Para ese tiempo se había trasladado a trabajar como profesor a la universidad de Marburg a raíz de problemas acaecidos con Robert Koch. Estableció contacto con Emile Roux y Paul Ehrlich, quienes le asesoraron en la producción a gran escala del antisuero y en la mejora de su calidad en vistas a su comercialización. Con estos mismos fines, se asoció con la compañía Hoechst y posteriormente decidió convertirse en empresario fundando su empresa en Marburg: la compañía Behringwerker, dedicada a la producción del antisuero y que se convirtió en la mayor productora en Alemania. Pero el gran éxito de la compañía vino con el desarrollo de la inmunización frente al tétanos durante la Primera Guerra Mundial. Las heridas severas de los soldados los hacía susceptibles a la infección de tétanos y para prevenir se les inyectaba el antisuero descubierto años anteriores por Emil Behring y producido a gran escala en su compañía.

Este destacado científico alemán recibió varios reconocimientos importantes por sus aportaciones a la medicina. En 1901 le fue otorgado el premio Nóbel en Fisiología y Medicina por el descubrimiento del antisuero contra la difteria; primer premio Nóbel otorgado en esta categoría. Emil Behring también recibió un título nobiliario, formando parte de la nobleza alemana como Emil "von" Behring. Además fue asesor Médico de Prusia, de la Legión de Honor francesa y miembro honorario de varias instituciones médicas europeas.

Muy relacionado con el ámbito militar y un gran cultivador de las relaciones sociales, Emil Behring supo involucrarse en aquellos ambientes que le facilitaron el trabajo y el desarrollo de su empresa. Fue una persona muy ambiciosa y de carácter autoritario. En el trabajo destacó por ser disciplinado y entusiasta. Aunque su forma de trabajar y su personalidad le generaron muchas críticas en el ámbito científico, fueron la clave para culminar plenamente su proyecto: desde el descubrimiento básico y la producción industrial, a la aplicación médica de la cura para la difteria y el tétanos. Sin duda, Emil Behring fue un pionero en su tiempo, un emprendedor que supo vincular el ambiente científico con el empresarial y así lograr el financiamiento necesario para dar continuidad a sus proyectos.

- Nobel Prize.org. The Official Website of the Nobel Prize. Emil Behring [Fotografía]. Recuperada de https://www.nobelprize. org/nobel_prizes/medicine/laureates/1901/behring-facts.html

\section{Otras fuentes}

- Bracha, A., \& Tan, S. Y. (2011). Emil von Behring (1854-1917): Medicine's first Nobel laureate. Singapore Medical Journal, 52(1), 1-2.

- Kaufmann, S. H. E. (2017). Emil von Behring: translational medicine at the dawn of immunology. Nature reviews. Immunology, 17(6), 341-343.

- Villanueva-Meyer, M. Emil von Behring (1854-1917): Pionero de la inmunología y descubridor de las vacunas contra el tétano y la difteria. Galenus, 37(1), 40-42, 2013. 